\title{
Hydrothermal Synthesis of Different Zinc Oxide Nanostructures: Growth, Structure and Gas Sensing Properties
}

\author{
Ping Wang ${ }^{1,2, *}$, Dong-yan Liu ${ }^{1, *}$ and Dong-sheng $\mathrm{Li}^{1}$ \\ ${ }^{1}$ College of Civil Engineering, Chongqing University, Chongqing, 400045, P. R. China \\ ${ }^{2}$ Department of Military Architecture and Civil Engineering, Logistical Engineering University of PLA, \\ Chongqing, 401331, P. R. China
}

\begin{abstract}
We report the nanostructures and gas-sensing properties of Zinc oxide nanosheets prepared by hydrothermal method. In particular, needlelike Zinc oxide was also prepared for comparison. The morphology and structural properties were investigated using X-ray diffraction (XRD), scanning electron microscope (SEM) and transmission electron microscope (TEM). Subsequent gas-sensing measurement between Zinc oxide nanosheets and Zinc oxide nanoaciculars indicated that the nanosheets exhibited higher gas response to ethanol. The enhanced gas sensing properties of Zinc oxide nanosheets suggests that governing the shape and the surface structure of nano Zinc oxide is a significant approach for developing high performance gas sensors. [doi:10.2320/matertrans.M2012203]
\end{abstract}

(Received May 21, 2012; Accepted August 29, 2012; Published October 11, 2012)

Keywords: nanosheets, ZnO, hydrothermal, gas sensor

\section{Introduction}

Zinc oxide $(\mathrm{ZnO})$, a direct wide band gap $(3.37 \mathrm{eV})$ semiconductor with a large excitation binding energy $(60 \mathrm{meV})$, is a typical multifunctional oxide ceramic materials possessing a suite of useful properties. ${ }^{1)}$ Especially in the applications of gas sensing, $\mathrm{ZnO}$ has attracted the affection of many researchers owning to its many advantages such as simple manufacture technique, low cost, rapid response and recovery time. ${ }^{2)}$ On the other hand, nanomaterials, due to their large surface area-to-volume ration, size effect and quantum, may own novel gas sensing properties. Therefore, great deals of efforts have been devoted into developing zinc oxide materials in nanometerscales for gas sensing applications. However, even in nanometer-scales, parameters such as dimensionality, morphology, crystal phase and crystallinity still have impacts on the gas sensing performances of metal oxide. ${ }^{3)}$ However, manipulating the shape and size of $\mathrm{ZnO}$ in a controllable way is still a great challengeable task. Many preparing methods such as thermal evaporation, ${ }^{4,5)}$ chemical vapor deposition $\left.(\mathrm{CVD})^{6}\right)$ are employed to synthetize nano and microstructure $\mathrm{ZnO}$ with various exciting shapes in the past few decades. Nevertheless, each of these preparation methods may have one or more shortages, such as being involved vacuum environment, complicated processes or high temperature, which may result in low crystallinity, impurity or poor dispersion. In contrast, the solution-based technique, which combines merits of easiness, economy and good operability for potential large-scale preparation, is considerably popular. $\mathrm{ZnO}$ single crystals with a variety of morphologies, including rods, tubes, disks, flowers, and other self-assembled architectures have been successfully synthesized by the typical solution approach. ${ }^{7-10)}$ In this paper, we employed a facile and tunable hydrothermal process to prepare $\mathrm{ZnO}$ nanosheets and nanoneedles.

*Corresponding author, E-mail: cquwangping@yahoo.com.cn
Furthermore, the crystal structure, morphologies, and formation conditions are elaborated, and latter gas sensing measurement indicates that $\mathrm{ZnO}$ nanosheets show higher gas response as compare to nanoneedles, thereby holding technological promise for fabricating excellent ethanol gas sensor.

\section{Experimental}

\subsection{Preparation of powder sample}

All of the chemical reagents were analytical grade and used without further purification. The detailed preparation process was as follows. Zinc acetate dehydrate $(5 \mathrm{mM})$, sodium citrate $(10 \mathrm{mM})$ and cetyltrimethylammonium bromide (CTAB, $10 \mathrm{mM}$ ) were dissolved into $50 \mathrm{ml}$ distilled water, followed by a stirring for $15 \mathrm{~min}$ using magnetic stirrer. Next, the solution was transferred into autoclaves and treated at $130^{\circ} \mathrm{C}$ for $12 \mathrm{~h}$ under autogenously pressure and then cooled down to room temperature naturally. The resulting white products were separated by centrifugation and washed with distilled water and ethanol for three times to remove unexpected ions, finally dried at $60^{\circ} \mathrm{C}$ overnight in air. Similarly, the $\mathrm{ZnO}$ nanoneedles were prepared according to Ref. 11), where zinc foil was used as raw material and butyl amine as agent and the hydrothermal condition is $140^{\circ} \mathrm{C}$ for $12 \mathrm{~h}$.

\subsection{Fabrication of sensor devices}

Firstly, a proper amount of the final powder was mixed with several drops of distilled water to form a paste. Then, the paste of powder was coated onto the $\mathrm{Al}_{2} \mathrm{O}_{3}$ tube (with a diameter of $1.2 \mathrm{~mm}$ ), which has two Au electrode wires that are separated at each end of the tube by $6 \mathrm{~mm}$. A Ni-Cr alloy filament which was inserted into the tube was used as heater, the temperature of coated tube could be controlled by regulating heating voltage. The coated ceramic tubes were finally sintered at $300^{\circ} \mathrm{C}$ for $2 \mathrm{~h}$, forming a covered sensing film with thickness of about $30 \mu \mathrm{m}$. 


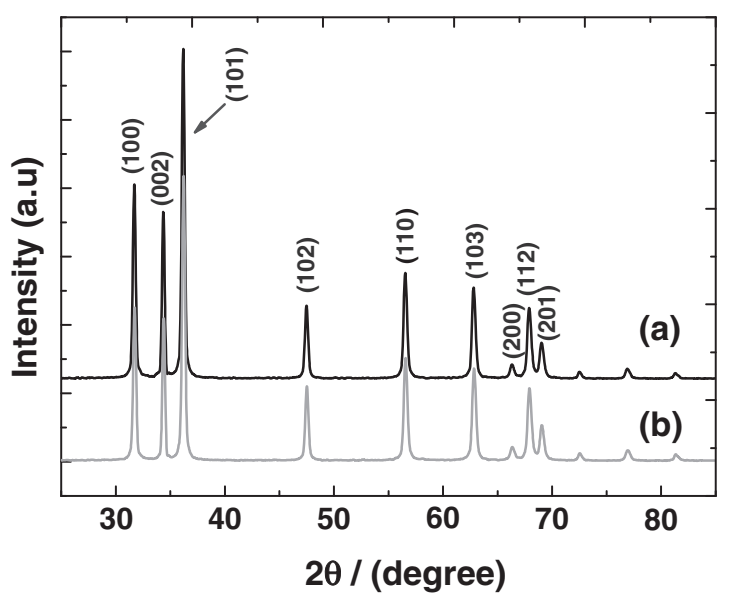

Fig. 1 XRD patterns of the products synthesized with different reagents: (a) zinc foil and butyl amine; (b) Zinc acetate dehydrate, sodium citrate and $\mathrm{CTAB}$

\subsection{Measurement}

Crystal structure of the samples was characterized by $\mathrm{X}$ ray diffraction (XRD) using RigakuD/Max-1200X diffractometry with the $\mathrm{Cu} \mathrm{K} \alpha$ radiation operated at $30 \mathrm{kV}$ and $100 \mathrm{~mA}$, surface morphologies of the samples were observed using a Hitachi S-4300 SEM, transmission electron microscope (TEM) images were obtained on a Hitachi H-600 with an accelerating voltage of $75 \mathrm{kV}$.

Electrical resistance in air and gas-sensing studies were carried out on a static system (HW-30A, Hanwei Electronics Co. Ltd., Henan Province, China) with the constant loop voltage of $5 \mathrm{~V}$ in laboratory condition $\left(30^{\circ} \mathrm{C}, 40 \%\right.$ relative humidity). In this paper, the response of sensor $(S)$ was defined as the ratio of resistance in air $\left(R_{\mathrm{a}}\right)$ to that in a target gas $\left(R_{\mathrm{g}}\right)$.

\section{Results and Discussion}

\subsection{Characteristic of sensing material}

To determine structure and chemical composition of the as-prepared products, we first performed XRD analysis. As shown in Fig. 1, the peaks of two kinds of prepared powder are all consistent with the diffraction patterns of the hexagonal wurtzite phase (space group $\mathrm{P} 6_{3} \mathrm{mc}$, JCPDS card file No. 36-1451) and no impure peaks can be found, where textural orientations of detected matters are given as well for easy reference. In addition, some of the diffraction patterns are shape and strong, signifying the high-degree crystallization of $\mathrm{ZnO}$ nanostructures.

The representative surface morphologies of the $\mathrm{ZnO}$ nanostructures were observed by SEM. Figures 2(a) and 2(b) illustrate SEM micrographs of the obtained samples prepared in the presence of butyl amine and sodium citrate + CTAB, respectively. The sample synthesized with butyl amine shows needle-like structure and arranged randomly. For a single nanoneedle, the two endpoints were specially poignant. Then we could see from Fig. 2(b) that numerous sheet-like nanostructures were obtained when sodium citrate and CTAB were used as assistant agents. Most of these nanosheets are grown interactively and mostly perpendicular to each other with many pores and intervals, which should play an important role in enhancing the sensing performances of $\mathrm{ZnO}$. Figure 2(c) is a TEM image of the sample prepared with butyl amine, clearly revealing their needle-like morphology. These nanoneedles have the maximum diameter less than $100 \mathrm{~nm}$ and length in micrometer level. From the TEM image of the sample prepared with sodium citrate and CTAB shown in Fig. 2(d), one can see that the irregular nanosheets adhere each other with the average thickness within $100 \mathrm{~nm}$.

The butyl amine, sodium citrate and CTAB play important roles in determining the $\mathrm{ZnO}$ morphology under current hydrothermal process. Based on the above experimental results, we considered some plausible reasons for the morphology evolution of the $\mathrm{ZnO}$ nanostructures. Although there are some different chemical reactions between the two hydrothermal processes, nucleation and crystal growth are still two main processes to form the different morphologies. $\mathrm{ZnO}$ nuclei could be formed when the concentration of $\mathrm{Zn}^{2+}$ and $\mathrm{OH}^{-}$reach the super saturation degree of $\mathrm{ZnO}$. The formed $\mathrm{Zn}(\mathrm{OH})_{2}$ also contributes to $\left[\mathrm{Zn}(\mathrm{OH})_{4}\right]^{2-}$ which is the growth unit of $\mathrm{ZnO} .^{12)} \mathrm{ZnO}$ is a well-known polar crystal consisting of numerous positive polar planes which are thermodynamically unstable, so the nucleation and growth tend to take place on these planes. The groups of $\left[\mathrm{C}_{4} \mathrm{H}_{9}\right]^{+}$, generated by butyl amine, can act as carriers via bonding with the precursors, promoting the growth rate of positive polar surface. Therefore, during subsequent growth, the (0001) facet can exhibit faster growth rate than other planes because of preferential adsorption in the presence of butyl amine, making (0001) facet to disappear at last while the facets grow slower will be retained, finally leading to the poignant shape at the (0001) end of the $c$-axis. ${ }^{11)}$ Therefore, the needle-like $\mathrm{ZnO}$ are formed easily. However, in the situation of formed nanosheets, the external environment is very different in the aqueous system. The obtained $\mathrm{ZnO}$ nanosheets are not uniform due to synchronous and slow growth on the polar planes. The $\mathrm{C}_{6} \mathrm{H}_{5} \mathrm{O}_{7}{ }^{3-}$ molecules of sodium citrate could also be adsorbed on the (0001) polar surface of $\mathrm{ZnO}$, but play a role in preventing its specific growth with reducing the higher surface energy. ${ }^{13)}$ Consequently, the random growth enhances the uniformity and promotes the sheet-like structure. Moreover, the CTAB plays a part in gathering these formed nanosheets to engender pores and intervals structures.

\subsection{Gas sensing properties}

To understand how the morphology of $\mathrm{ZnO}$ affect their gas sensing properties, we investigated the gas sensing properties of $\mathrm{ZnO}$ nanosheets and nanoneedles towards reducing ethanol gas. Figure 3 represents the dynamic responserecovery curves of $\mathrm{ZnO}$ samples to ethanol gas. One can see that the sensors not only can recover to initial resistances, but also show very considerable response-recovery capabilities. Moreover, during the time slots 40 to $80 \mathrm{~s}$ and 160 to $200 \mathrm{~s}$, it is apparently that $\mathrm{ZnO}$ with sheet-like morphology show higher response than that of needle-like morphology.

It is accepted that, upon the sensor is exposed to air, the oxygen molecules in air will be adsorbed on the surface and grain boundaries of sensor. Then adsorbed oxygen on the surface would trap the electrons in the semiconductor 

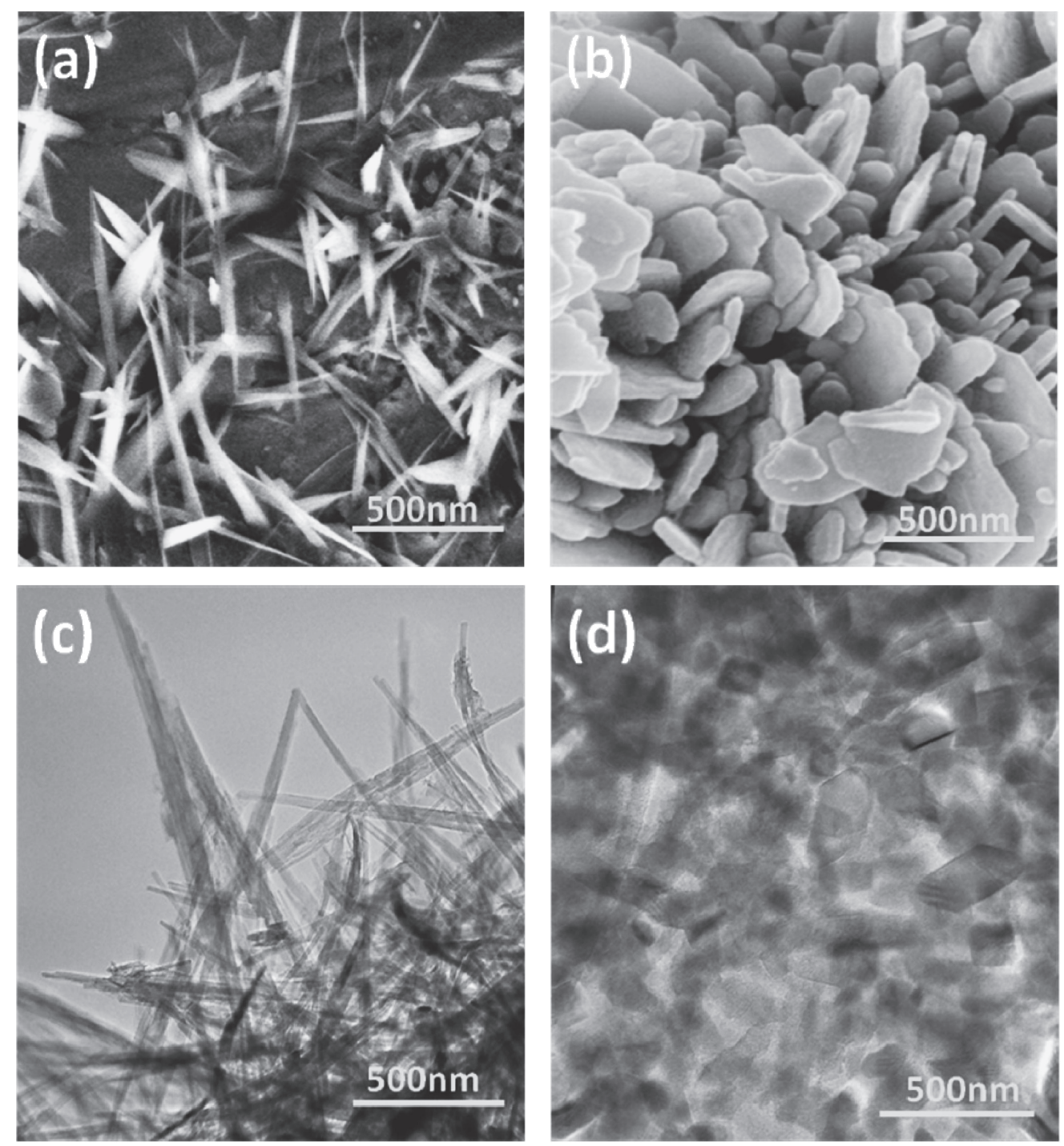

Fig. 2 SEM images ZnO nanostructures prepared with different reagents: (a) zinc foil and butyl amine; (b) zinc acetate dehydrate, sodium citrate and CTAB. Corresponding TEM images are shown in (c) and (d).

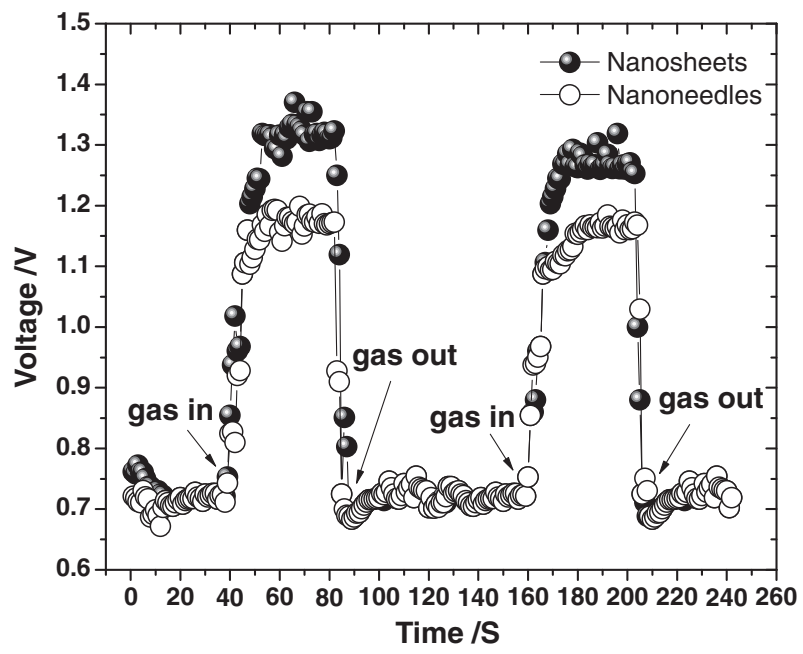

Fig. 3 The dynamic response-recovery curves of the samples toward ethanol gas.

conduction band to form chemisorbed oxygen species including $\mathrm{O}_{2}^{-}, \mathrm{O}^{-}$or $\mathrm{O}^{2-}$, etc., resulting in a thick electro depletion layer, as the following reactions happened:

$$
\begin{gathered}
\mathrm{O}_{2}(\mathrm{~g}) \rightarrow \mathrm{O}_{2} \text { (ads) } \\
\mathrm{O}_{2} \text { (ads) }+\mathrm{e}^{-} \rightarrow \mathrm{O}_{2}^{-} \text {(ads) } \\
\mathrm{O}_{2}{ }^{-} \text {(ads) }+\mathrm{e}^{-} \rightarrow 2 \mathrm{O}^{-} \text {(ads) }
\end{gathered}
$$

Therefore, the concentration of electrons in the conduction band would decrease and the resistance of the material would increase. When the reducing gas such as ethanol is introduced to the sensor, a chemical reaction would occur between $\mathrm{C}_{2} \mathrm{H}_{5} \mathrm{OH}$ and $\mathrm{O}^{2-}$ (ads) on the surface of $\mathrm{ZnO}$. The possibility of reaction can be explained as:

$$
\begin{gathered}
\mathrm{C}_{2} \mathrm{H}_{5} \mathrm{OH}(\text { gas })+\mathrm{O}^{2-}(\text { ads }) \rightarrow \mathrm{C}_{2} \mathrm{H}_{5} \mathrm{O}^{-}(\text {gas })+\mathrm{OH}^{-}(\text {ads }) \\
\mathrm{C}_{2} \mathrm{H}_{5} \mathrm{O}^{-}(\text {gas }) \rightarrow\left(\mathrm{C}_{2} \mathrm{H}_{5}\right)_{2} \mathrm{O}(\text { ads })+\mathrm{O}^{-}(\text {ads })+\mathrm{e}^{-} \\
\mathrm{C}_{2} \mathrm{H}_{5} \mathrm{OH}(\text { gas })+\mathrm{O}^{2-}(\text { ads })+\text { hole } \rightarrow \mathrm{CO}_{2}+\mathrm{H}_{2} \mathrm{O}+\mathrm{V}_{0}
\end{gathered}
$$

Where $\mathrm{V}_{0}$ is a doubly charged oxygen vacancy, electrons produced from the reaction would decrease the resistance of the material, result in the output voltage increased when the gas sensor exposed in the testing gas. ${ }^{14-17)}$

The quick and higher response of $\mathrm{ZnO}$ nanosheets should be ascribed to its pores, intervals structures and more relatively unstable $\mathrm{Zn}$-terminated $\mathrm{ZnO}$ (0001) polar surfaces. 
For a film gas sensor, gas diffusion is a significant factor that effecting the sensor response and response time. The porous structure of nearly vertically adhered nanosheets not only can make the gas reach the deep layer quickly, but also add the surface area-to-volume ration. In general, the larger surface area-to-volume ration, the more excellent gas sensing performance. On the other hand, the nanosheets exist much more unstable Zn-terminated $\mathrm{ZnO}$ (0001) polar surfaces which are chemically active with the $\mathrm{O}_{2}$ gas phase, increasing interaction with ethanol gas. ${ }^{18)}$ Therefore, the response of $\mathrm{ZnO}$ nanosheets is higher than nanoneedles.

\section{Conclusions}

In summary, the $\mathrm{ZnO}$ nanostructures with sheet-like morphology were successfully synthesized by facile hydrothermal process. It was found that sodium citrate and CTAB have significant morphological directing function. For comparison, needle-like $\mathrm{ZnO}$ was also prepared. Subsequent gas measurement indicated that the $\mathrm{ZnO}$ nanosheets behave higher response than nanoneedles, which was attributed to the porous structure and more $\mathrm{Zn}$-terminated $\mathrm{ZnO}$ (0001) polar surfaces in nanosheets. This kind of sheet-like structure provides a new approach for rational design and development of sensing materials.

\section{REFERENCES}

1) T. Alammar and A. Mudring: Mater. Lett. 63 (2009) 732.

2) Y. Zeng, T. Zhang and L. Qiao: Mater. Lett. 63 (2009) 843.

3) H. Zhang, T. Liu, L. Huang, W. Guo, D. Liu and W. Zeng: Physica E 44 (2012) 1467.

4) B. Yao, Y. Chan and N. Wang: Appl. Phys. Lett. 81 (2002) 757.

5) M. Huang, Y. Wu, H. Feick, N. Tran, E. Weber and P. Yang: Adv. Mater. 13 (2001) 113.

6) Y. Kashiwaba, K. Sugawara, K. Haga, H. Watanabe, B. Zhang and Y. Segawa: Thin Solid Films 411 (2002) 87.

7) H. Zhang, D. Yang, S. Li, X. Ma, Y. Ji, J. Xu and D. Que: Mater. Lett. 59 (2005) 1696.

8) L. Greene, M. Law, D. Tan, M. Montano, J. Goldberger, G. Somorjai and P. Yang: Nano Lett. 5 (2005) 1231.

9) B. Liu and H. Zeng: J. Am. Chem. Soc. 125 (2003) 4430.

10) W. Guo et al:: Sens. Actuators B: Chem. (2012) doi:10.1016/ j.snb.2012.02.093.

11) T. You, J. Yan, Z. Zhang, J. Li, J. Tian, J. Yun and W. Zhao: Mater. Lett. 66 (2012) 246.

12) R. S. Yadav and A. C. Pandey: Physica E 40 (2008) 660.

13) W. Guo, T. Liu, W. Zeng, D. Liu, Y. Chen and Z. Wang: Mater. Lett. 65 (2011) 3384.

14) W. Zeng, T. Liu and Z. Wang: J. Mater. Chem. 22 (2012) 3544.

15) W. Zeng, T. Liu, D. Liu and C. Lv: Physica E 44 (2011) 37.

16) W. Zeng and T. Liu: Physica B 405 (2010) 1345.

17) W. Zeng, T. Liu and Z. Wang: Physica E 43 (2010) 633.

18) W. Guo, T. Liu, L. Huang, H. Zhang, Q. Zhou and W. Zeng: Physica E 44 (2011) 680. 